how alcohol habits change with age and in different subgroups. The aim is to study stability and variations in alcohol habits for demographic subgroups over time.

Methods The data derives from the longitudinal population-based study of mental health (the PART-study) in Stockholm County, Sweden. The data were gathered using postal questionnaires and register data, including three measure points 1998-2000 $\left(\mathrm{n}_{\mathrm{t} 1}=10441\right), 2000-2003\left(\mathrm{n}_{\mathrm{t} 2}=8613\right.$ persons), and 2010 (tentative $\mathrm{n}_{\mathrm{t} 3}=5776$ ) with ages ranging between 20 and 64 years at $\mathrm{t}_{1}$. The Alcohol Use Disorders Identification Test (AUDIT) was used to measure alcohol habits. Intra-class correlations over the three time points are used as measures of stability, calculated from the AUDITscores for different subgroups.

Results The results will be presented for subgroups, comparing the intra-class correlations. The findings and the contextualisation of the results will be discussed for the subgroups with stabile and high variation in alcohol habits.

Conclusion While a vast number of studies have described the stability of alcohol habits for whole cohorts, the literature on stability and variation in specific subgroups is sparse. The findings will add to the knowledge about alcohol habits in demographic subgroups.

\section{P1-528 STATISTICAL MODELS IN LONGITUDINAL EVALUATION OF CHANGES IN HEALTH BEHAVIOURS}

doi:10.1136/jech.2011.142976h.16

K Szamotulska, ${ }^{*}$ E Mierzejewska. National Research Institute of Mother and Child, Warsaw, Poland

Introduction During long-lasting intervention program, the sociodemographic structure of the target population may change and influence the results of the overall evaluation. The Primary Prevention Program of Neural Tube Defects in Poland which was carried out in 1997-2007 in the whole country, gives the opportunity to discuss application of different statistical models in this context.

Methods Three surveys were conducted on representative samples of women aged $18-35$ years during the Program activity. The samples were drawn from patients of the same randomly selected primary health centres in five chosen main administrative regions in 2001 $(n=775), 2003 \quad(n=756)$ and $2007 \quad(n=756)$. Relative changes in women's knowledge and behaviour concerning folic acid were analysed by Poisson regression and generalised estimating equation models.

Results The proportion of women taking folic acid during the pregnancy increased from $52 \%$ in 2001 to $68 \%$ in 2003 and $86 \%$ in 2007 and the proportion of women beginning supplementation before the pregnancy increased from $11 \%$ to $15 \%$ and $29 \%$, respectively. The behaviours towards folic acid strongly differentiated according to socio-demographic characteristic, which was also not stable during the period of program implementation. For example, the proportion of post-secondary educated women increased from $20.4 \%$ in 2001 to $23.1 \%$ in 2003 and $41.7 \%$ in 2007 . These changes influenced slightly, although not significantly and depending on statistical approach chosen, the overall magnitude of effects.

Conclusions Time-depending socio-demographic structure of the target population has to be considered in evaluation of health promotion programs.

\section{P1-529 THE RELATIONSHIP BETWEEN THE WEIGHT IN EARLY CHILDHOOD AND THE FOLLOWING GROWTH CURVE IN WEIGHT}

doi:10.1136/jech.2011.142976h.17

${ }^{1} \mathrm{H}$ Takahashi, ${ }^{2} \mathrm{~T}$ Mikami, ${ }^{3} \mathrm{M}$ Yoshinaga, ${ }^{4} \mathrm{M}$ Shinomiya, ${ }^{5} \mathrm{~A}$ Miyazaki. ${ }^{1}$ Department of Epidemiology, Graduate School of Comprehensive Human Sciences, University of
Tsukuba, Tsukuba, Ibaraki, Japan; ${ }^{2}$ School of Medical Sciences, University of Tsukuba, Tsukuba, Ibaraki, Japan; ${ }^{3}$ Department of Pediatrics, National Hospital Organization Kagoshima Medical Center, Kagoshima, Japan; ${ }^{4}$ Chiba Medical Association, Chiba, Japan; ${ }^{5}$ Department of Pediatrics, Shakaihoken Takaoka Hospital, Takaoka, Toyama, Japan

Introduction To prevent the young overweight is an important public health issue for healthy growth and the avoidance of future obesity-related diseases. However, the growth pattern varies among individuals, and it is little known whether or not the patterns depend on the weight in early childhood. The objective of this study is to clarify it.

Methods Subjects were 913 students of 15-18 years old (396 males and 517 females) of high schools in three prefectures in 2006-2008. Height and weight at birth, their 1.5, 3, 6, 9, 12 years and the current were obtained with a questionnaire asked to refer to mainly one's maternal and child health handbook. BMI z-score was defined as (one's BMI minus the mean BMI in population) divided by its population SD. Sex-specific latent growth curve models consisting of 2, 3 and 4 groups with up to the third power for the curve fitting were estimated by using seven BMI-scores at the ages, and the relationship of the weight status at birth and 1.5 years old to the curves were tested with the statistical software SAS 9.1.3.

Results Distinguished patterns were found for all the group models in males and only the 2 group model in female. The weight at birth and 1.5 years was significantly related to all the patterns in males, and to only the patterns of the 2 group model in female.

Conclusion The weight in early childhood related to the growth patterns in weight.

\section{P1-530 BLOOD PRESSURE CATEGORIES AND LONG-TERM MORTALITY RISK OF TOTAL AND COMPONENTS OF CARDIOVASCULAR DISEASES AMONG JAPANESE: A 24 YEAR FOLLOW-UP OF REPRESENTATIVE JAPANESE (NIPPON DATA80)}

doi:10.1136/jech.2011.142976h.18

${ }^{1} \mathrm{~N}$ Takashima, ${ }^{*}{ }^{1} \mathrm{~K}$ Miura, ${ }^{1} \mathrm{~T}$ Ohkubo, ${ }^{2} \mathrm{~T}$ Okamura, ${ }^{1,3} \mathrm{~T} \mathrm{C}$ Turin, ${ }^{1} \mathrm{Y}$ Murakami, ${ }^{1,4} \mathrm{~N}$ Okuda, ${ }^{1} \mathrm{~A}$ Fujiyoshi, ${ }^{1} \mathrm{~T}$ Kadowaki, ${ }^{1} \mathrm{~S}$ Nagasawa, ${ }^{1} \mathrm{~A}$ Kadota, ${ }^{1} \mathrm{Y}$ Kita, ${ }^{4} \mathrm{~A}$ Okayama, ${ }^{1} \mathrm{H}$ Ueshima. ${ }^{1}$ Shiga University of Medical Science, Otsu, Japan; ${ }^{2}$ Keio University, Tokyo, Japan; ${ }^{3}$ University of Calgary, Calgary, Canada; ${ }^{4}$ Japanese AntiTuberculosis Association, Tokyo, Japan

In Western population, blood pressure (BP) was reported to predict long-term (over 20 years) future mortality of cardiovascular diseases (CVD). However, there is few reports based on long-term follow-up studies in Asian population where stroke is dominant among CVDs. We investigated the association between $\mathrm{BP}$ and 24 year mortality risk of total and components of CVD in a representative Japanese population. Methods A cohort study of nationwide representative Japanese samples, a total of 8592 men and women age 30 and over without history of CVD and anti-hypertensive medication was followed for 24 years (mean follow-up, 21.3 years). Multivariate-adjusted HRs of total and components of CVD death according to the BP categories (Seventh Report of the Joint national Committee criteria) were calculated using the Cox proportional hazard model.

Results During the follow-up period, 689 participants died from CVD. Multivariate-adjusted HR of total and components of CVD mortality was progressively and significantly greater from the lowest BP group. Compared with normal BP, adjusted HRs in stage 2 hypertension was 2.45 for total CVD death and 5.99 for cerebral haemorrhage death. An adjusted HR for total CVD were higher in younger participants aged 30 to 59 years than that in elder aged 60 years and over at baseline.

Conclusion Blood pressure in general Japanese retain a strong association with total and components of CVD death during next 24 years. The association was steeper in younger participants. 